\title{
Enhanced Performance of SVC via Using Rotor Speed Deviation Signal (RSDS)
}

\author{
Hamidreza Ghorbani ${ }^{1}$, Mehdi Monadi ${ }^{1}$, Alvaro Luna ${ }^{1}$, Jose Ignacio Candela ${ }^{1}$, Pedro Rodriguez ${ }^{1,2}$ \\ ${ }^{1}$ Universitat Politechnica de Catalunia (UPC)-Barcelona Tech, \\ Rambla Sant Nebridi \\ Terrassa, Barcelona (Spain) \\ Tel.: +34 937398549 \\ ${ }^{2}$ Abengoa Research, Spain \\ Emails: ghorbani.h1979@gmail.com,mehdi.monadi@estudiant.upc.edu,luna@ee.upc.edu, \\ candela@ee.upc.edu,prodriguez@ee.upc.edu
}

\section{Acknowledgements}

This work was supported in part by the Spanish Ministry of Economy and Competitiveness under Project ENE2013-48428-C2-2-R. Any opinions, findings, and conclusions or recommendations expressed in this material are those of the authors and do not necessarily reflect those of the host institutions or founders.

\section{Keywords}

Static Var Compensator (SVC), Rotor Speed Deviation Signal (RSDS), Transient stability.

\begin{abstract}
Transient stability enhancement through using a Static Var Compensator (SVC) and Rotor Speed Deviation Signal (RSDS) of synchronous machine are thoroughly investigated in this paper. Speed deviation signal of generator as a remote signal is sent to SVC in order to achieve more transient stability. Studies are carried out on Kundur's four machine two-area test system which SVC is implemented on midway of transmission system. The simulation results verify the effectiveness of the proposed method to improve rotor angle stability versus different kinds of disturbances scenarios. The standard benchmark model is used for the analysis in the MATLAB/Simulink environment.
\end{abstract}

\section{Introduction}

Since 1960s, electromechanical oscillations have been observed when power networks are interconnected through weak tie lines [1]. Regarding economic and environmental considerations, electric power networks are developing and system damping is weakened. Low frequency oscillations occur more often than before, and insufficient damping of electromechanical oscillations is going to apparent significant problem for transmitted power. Power system stabilizers (PSSs) has major role to enhance the system damping. In some issues, when use of PSS cannot provide sufficient damping for inter-area oscillations, controllers of FACTS devices are alternative effective solutions [2]. Because these devices with supplementary controllers aiming at the improvement of dynamic conditions and transient stability. Therefore the usage of them in new electrical power systems is expanded.

PSSs and FACTS devices individually are appropriate solutions to eliminate power system oscillations and both of them are fast acting power system instruments. Practically several FACTS devices and PSSs have been installed in real power networks $[3,4]$ they have the potentials of interaction with each other to improve overall system dynamic performance as well.

Following a transient disturbance (such as faults on transmission circuits, transformers, buses), speed of synchronous generators and their angles will change instantly in various points of the power system. Stability depends on the severity of the disturbance and fault cleaning time, so if the subjected disturbance 
be cleared in proportional time system will be remained in stability condition [5]. Study period for transient stability studies is generally limited to 3 to 5 seconds following the disturbance and during this period of time mechanical power of generators are assumed constant.

From transient stability viewpoint worst kind of perturbation is three lines to ground (LLL-G) short circuit (symmetric) and worst fault location is first of transmission system that it is close to generators or power plant side. So in order to make certitude of the system ability to remain in stability condition worst case is considered in this study.

Static Var Compensator (SVC) is a set of electrical devices for providing fast-acting reactive power on transmission power networks. SVCs are part of the FACTS device family [6]. Using FACTS devices adjust the transferred power and this phenomenon causes to improve power system stability issues.

Here a brief has been done on the using of FACTS devices along power plant side controllers. Refs $[7,8]$ demonstrated a coordinated excitation and SVC controller for both transient stability and voltage regulation enhancement. But the authors just have studied on single machine system benchmark and their achievements have not been thoroughly involved on multi-machine power system studies and inter-area oscillation problems. In addition, Gibbard [9] presented the conventional power system stabilizer that provides appropriate damping performance in a wide range of system loading conditions. Various loading conditions in Ref. [10], is presented in order to analyze of robust design of conventional power system stabilizers in multi-machine power systems with using genetic algorithm. Bazanella et al. [11] demonstrated a method for coordinated design of PSSs and supplementary controllers for FACTS devices. The offered robustness control method is evaluated against severe but slow changes in operating conditions and it is not involved for fast dynamic transient conditions. As well as Refs $[12,13]$ develop new design procedures for simultaneous coordinated designing of FACTS damping controller and PSS. The offered controllers have been tested on multi-machine power systems through the simulation studies in order to show the damping enhancement of the inter-area oscillations.

This paper presents a survey on using of the Rotor Speed Deviation Signal (RSDS) in SVC controller. In this effort with using remote signal technology and transformation RSDS of synchronous generators into the center of SVC controller, rotor angle stability is enhanced. Recently using from remote signal and its transformation via satellites systems or optical fiber is more considered. In Ref [14], the mentioned technic with aiming Voltage Source Controller (VSC) to mitigate Sub-Synchronous Resonance (SSR) oscillations have been done. Majumder et al. In Ref. [15] has been presented a control method to handle time delays encountered in transmitting the remote signals in electrical networks.

Recently various approaches are offered to mitigate multi-machine power system oscillations therefore it seems elimination of inter-area oscillation is one of the main concerns for improving power system stability among researches $[16,17]$.

\section{Test System Modeling}

\section{Power system modeling}

The depicted test system in Fig .1 is based on the system consists of two areas which both areas are connected together through a weak tie and the considered SVC is implemented on midway of one of transmission lines. Each area consists of two coupled synchronous generator units, rated power of each synchronous generator is $835 \mathrm{MW}$ and their nominal voltages are $230 \mathrm{KV}$. The transmitted active power along the tie line is $413 \mathrm{MW}$. Parameters of the generator are listed in $[5,18]$. In order to increase the practicality, a hydrolic turbine and governor system is also considered in the simulations. The model used for this system is an IEEE standard model expressed in [19]. 


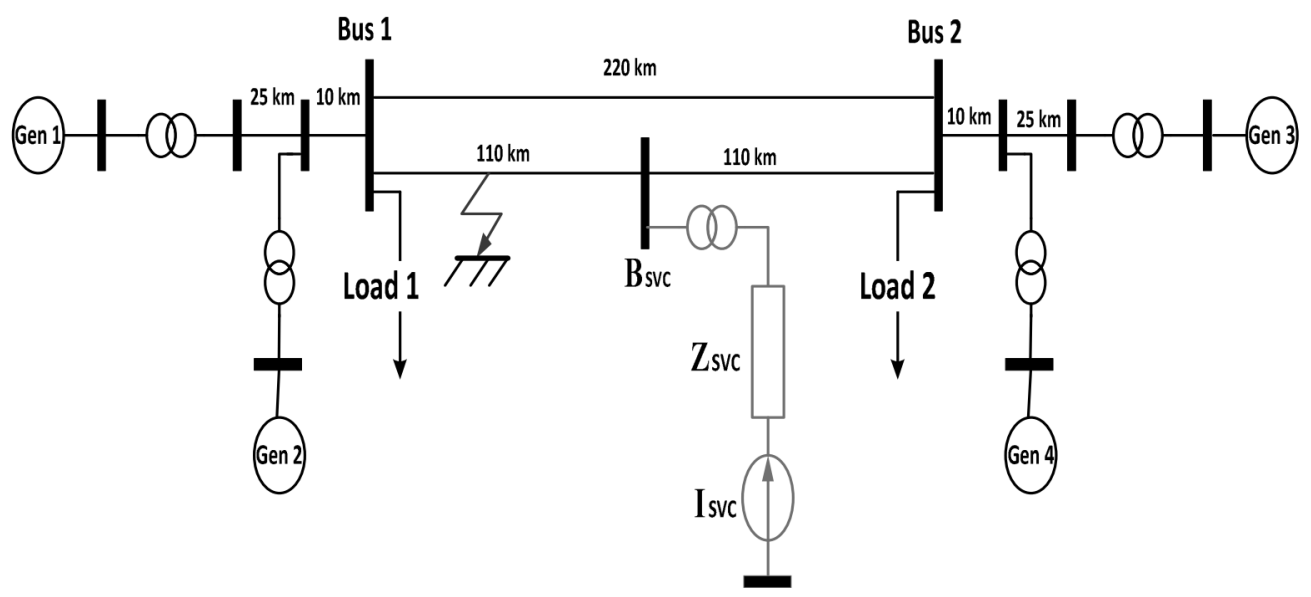

Fig.1. Standard four machine two-area system with implemented SVC

This system as an appropriate benchmark in order to analyze Low Frequency Oscillations (LFO) in large interconnected power networks is considered.

\section{SVC model}

The SVC is a shunt device of the FACTS family which it improves transient stability on power networks. As well as it regulates voltage by generating or absorbing reactive power. The SVC generates reactive power if voltage of the network is reducing also in case of increasing voltage profile at terminals the SVC absorbs reactive power. Injection of reactive power (capacitive or inductive reactive power) to grid is provided by switching three-phase capacitor banks and inductor banks connected on the secondary side of a coupling transformer. Each capacitor bank is switched on/off by three Thyristor Switched Capacitor (TSC). Reactors are either switched on/off phase-controlled (Thyristor Controlled Reactor or TCR). The considered SVC contains one 100 Mvar TCR bank and two 50 Mvar TSC banks which are connected to the middle of line 2 via a $230 \mathrm{kV} / 16 \mathrm{kV}$ (Yg/d), 340 MVA transformer, but in fig.2 is illustrated just one TSC bank and one TCR bank. All of three-phase banks (TSCs and TCR) are connected as delta so, during normal operation the zero-sequence tripplen harmonics (3rd, 9th,..) remain trapped inside the delta, thus reducing harmonic injection into the grid. Switching the TSCs allows a step change variation of the secondary reactive power (between 0-100 Mvar capacitive at $16 \mathrm{kV}$ ) by steps of 50 Mvar, as well as phase control of the TCR applies a continuous variation from zero to 100 Mvar inductive depending on system need [20,21].

\section{SVC control system}

SVC control system with RSDS is presented in Fig. 3. Positive sequence of primary voltage magnitude Vabc (Prim), is measured by voltage measurement unit and then is compared with VRef. Measurement unit is driven by Phase Locked Loop (PLL) in order to take into account frequency variation of the grid. On the other hand, RSDS (which it is getting from generator1 (G1)) with appropriate amplifier (KP) and passing from washout filter is added to the output of measurement unit. Finally the obtained error passes through a PI regulator presents the primary susceptance Bsvc. Distribution unit uses the computed Bsve to determine the TCR firing angle ( $\alpha$ ) and the status (on/off) of the two TSC branches.

RSDS has been used already as an essential part of PSS structure therefore the signal as an auxiliary signal is available to apply on SVC controller. 
The firing angle ( $\alpha$ ) as a function of the TCR susceptance (BTCR) is extracted from (1):

$$
B_{T C R}=\frac{2(\pi-\alpha)+\sin (2 \alpha)}{\pi}
$$

Firing unit includes of three independent subunits that each one of them consists of a PLL synchronized on line-to-line secondary voltage and a pulse generator for each of TCR and TSC sections. The pulse generator is feeding by the firing angle $\alpha$ and the TSC status which they are receiving from the distribution unit.

So, regarding to the above-mentioned descriptions, the control system is included:

1) Voltage measurement unit that it is measuring the positive-sequence voltage and it is programmable.

2) Voltage regulator that it uses the error magnitude (difference between the measured voltage Vm, RSDS and the reference voltage VRef) for concluding the required SVC susceptance to maintain the system voltage constant.

3) Distribution unit that determines the TSCs contribution rate which it should be switched in/out, and computes the firing angle $\alpha$ of TCRs.

4) Synchronizing system using a phase-locked loop (PLL) synchronized on the secondary voltages and a pulse generator that send appropriate pulses to the thyristors [21].

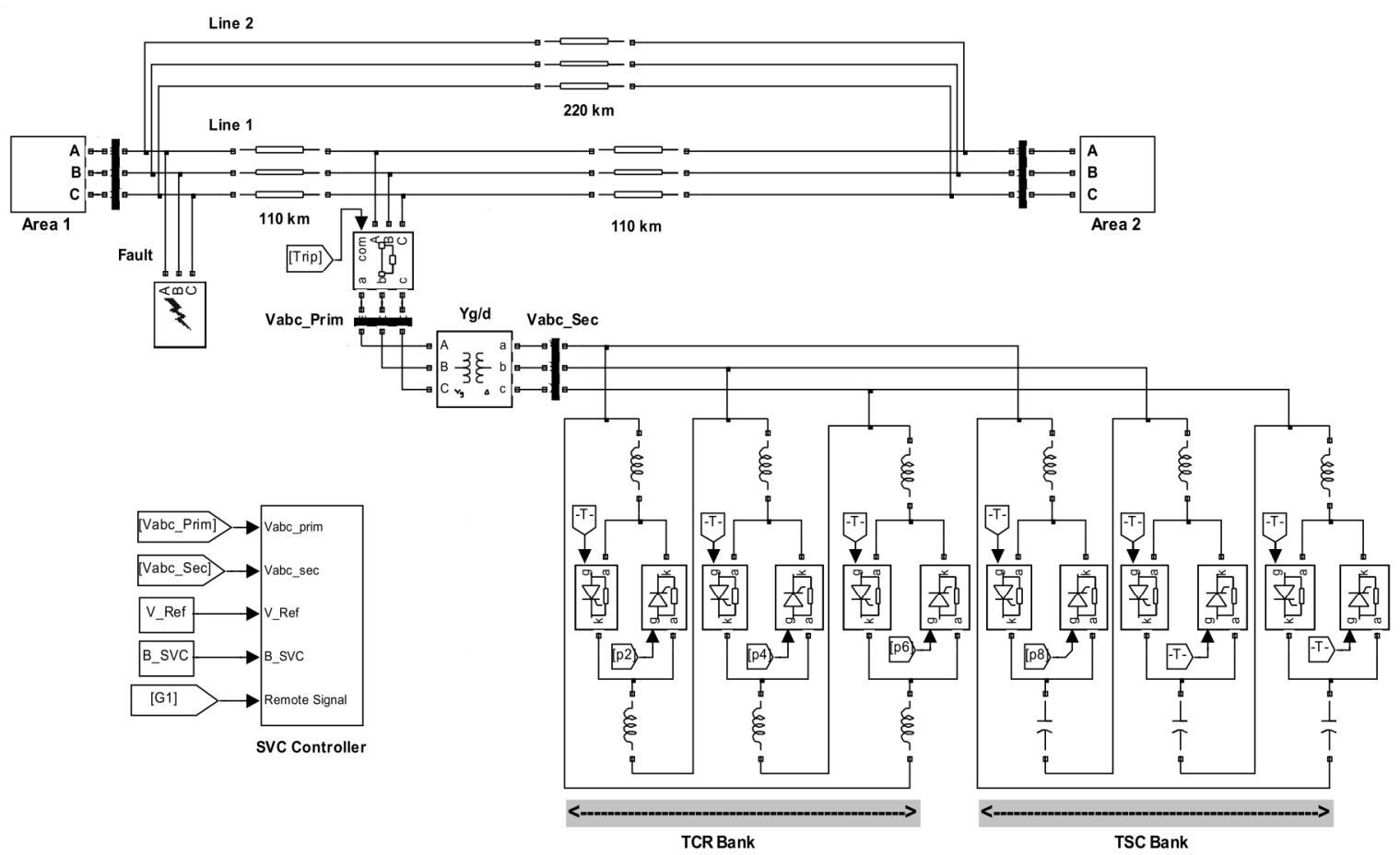

Fig.2 The modeled system with SVC in MATLAB environment 


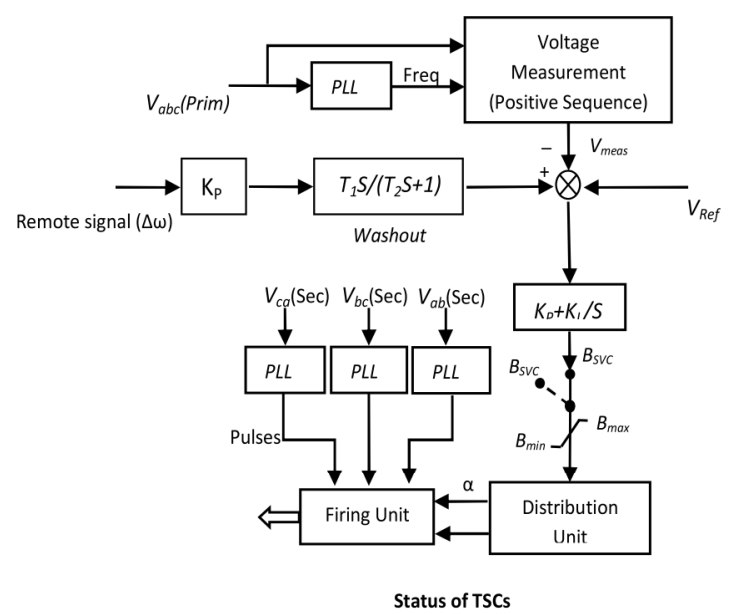

Fig.3 SVC control system

\section{Delay on Communication}

One of the most important issues in the wide-area-based control methods is the role of the delay of data transferring by communication on the performance of the method. This delay depends on several factors such as: distance between sending and receiving nodes and the protocol of data transmission [22]. Fig.4. shows the overall scheme of the proposed wide-area control.

On the other hand the main sources of the delay on communication can be categorized in; 1) time delay of change the measured values to a signal which can transfer by communication link; 2) time delay of data transferring between the nodes, i.e. sending and receiving substations; 3) time delay of change the transferred signals to values which are applicable to the proposed controller [23].

The speed of data transferring through communication media can be assumed to be close to the speed of light. Therefore, for a transmission line with several hundred kilometer length, the communication delay can be assume to be in the range of milliseconds. Similar to the method used in [24], in the following simulations of this paper assumed that the communication delay in $3 \mathrm{~ms}$.

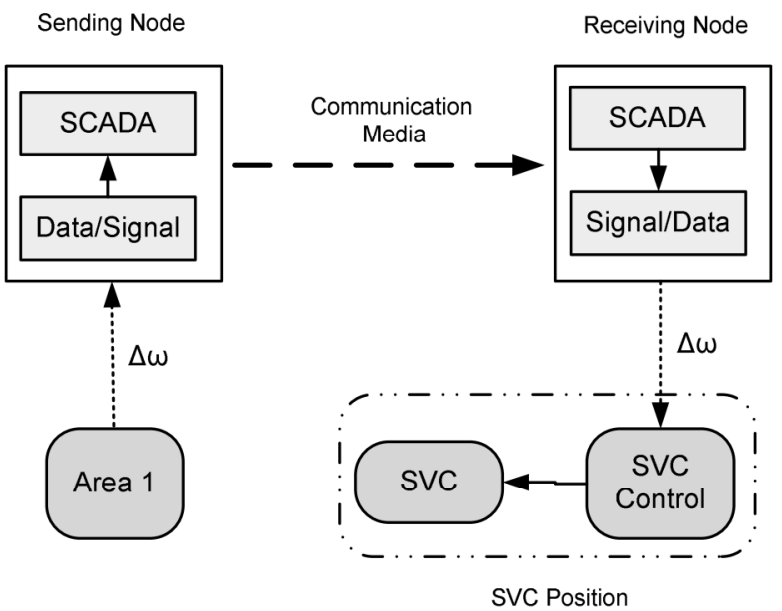

Fig. 4. The communication layout of the proposed control. 


\section{Simulation Results}

The simulations were carried out, in Sec.1 a three phase to ground (LLL-G) short circuit is assumed. This disturbance is occurred for seven cycles. Rotor angles three generators (G1, G2 and G3) are depicted in fig.5 (G4 angle is considered as a base angle). Regarding to the results using auxiliary signal causes to improve transient stability. As well as it damps more fast the oscillation of generator rotors so the settling time is improved.

In Fig.5 the dashed curves show system behavior in transient condition when remote signal is considered in SVC controller. As depicted results moreover transient stability improvement of subjected generator (G1) which is used its rotor speed deviation as the remote signal, this method causes to enhance other generators stabilities as well.

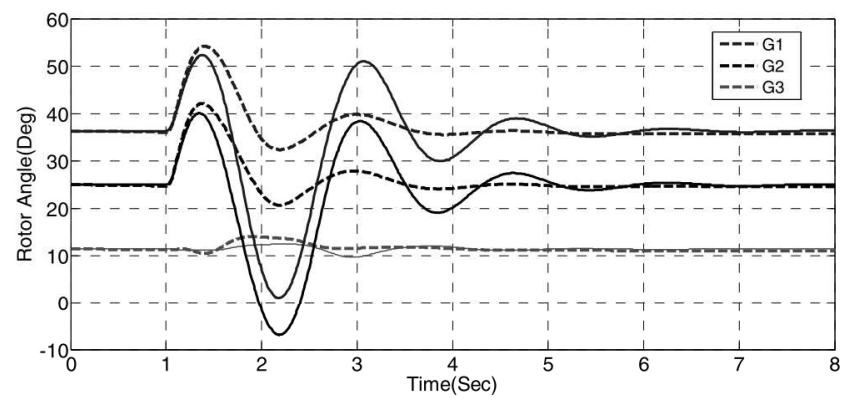

Fig. 5 Generators rotor angles (G1, G2, G3) in LLL-G fault condition.

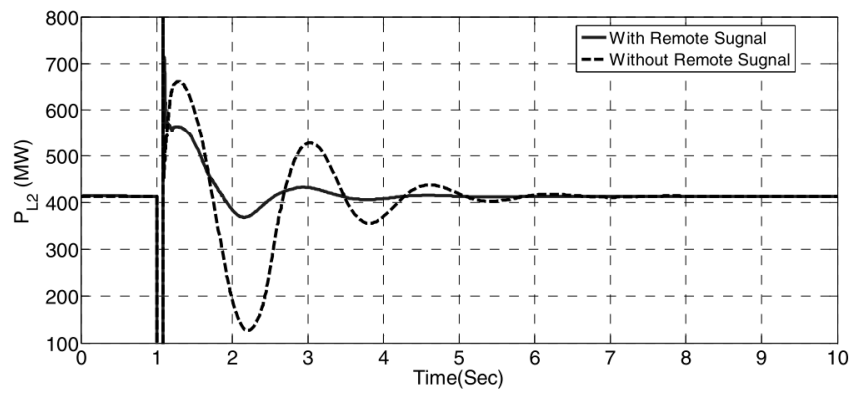

Fig. 6 Transmitted active power (MW).

In Fig.6 is shown the transmitted active power between areas under transient condition with and without presence of remote signal. As depicted simulation result in fig.6, using auxiliary signal in SVC controller enhanced quality of the transmitted active power and damped the appeared oscillations as well.

Generators rotor angle for same transient condition with assumption using rotor speed deviation of generator 2 is illustrated in fig.7 in order to have a comparison both of states is depicted in the figure. Regarding to the results in this case like previous state application of remote signal has improved the system transient stability.

Fig.8 shows the simulation results of lint to ground (L-G) fault which this kind of disturbance is considered for 8 cycles. 


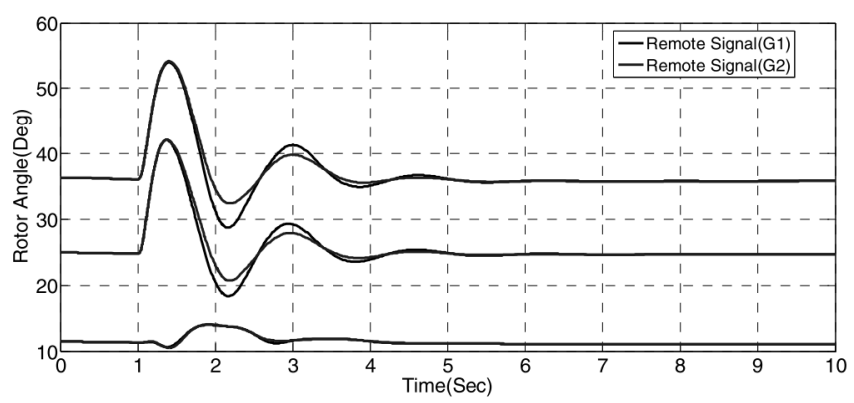

Fig. 7 Generators rotor angles (G1, G2, G3) in LLL-G fault condition

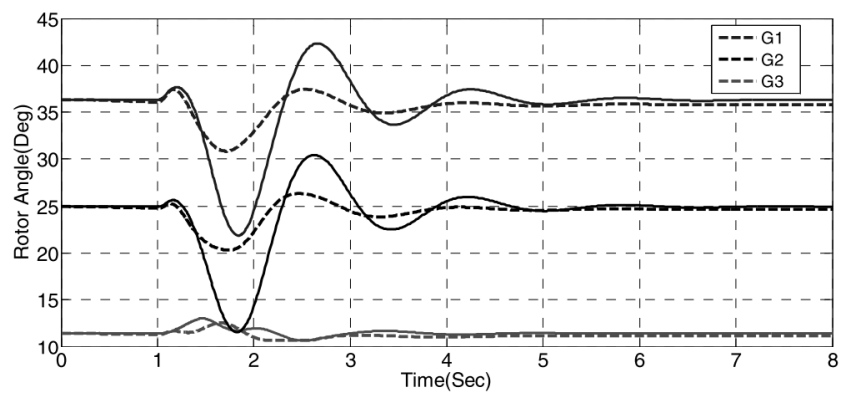

Fig. 8 Generators rotor angles (G1, G2, G3) in L-G fault condition

Like previous fault scenario the fault location is implemented on first of transmission line. As demonstrated, application of implemented auxiliary controller on SVC controller and using RSDS has enhanced the system transient stability.

\section{Conclusions}

Application of auxiliary stabilizer signals such as speed deviation of synchronous machine signals and sending them to control centres of power systems through optic fibre or communication links cause to make more stability in power systems.

In this paper is tried to show that using speed deviation of synchronous machine as a remote signal and sending the measured magnitude to implemented SVC controller in middle of transmission system has considerable effect to improve transient stability of the power system.

The investigated method moreover enhancing transient stability of subjected generator, also it causes to improve transient stability and rotor angle stability of other existence generators.

\section{References}

[1] M.A. Abido, Y.L. Abdel-Magid, "Coordinated design of a PSS and an SVC-based controller to enhance power system stability” International Journal of Electrical Power \& Energy Systems, Volume 25, Issue 9, pp. 695-704 November 2003

[2] X.Y. Bian, C.T. Tse, J.F. Zhang, K.W. Wang "Coordinated design of probabilistic PSS and SVC damping controllers" International Journal of Electrical Power \& Energy Systems, Volume 33, Issue 3, March 2011.

[3] Hammad AE. Analysis of power system stability enhancement by static VAR compensators. IEEE IEEE Transactions on Power systems, 1986;1(4):222-7.

[4] Padiyar KR, Varma RK. Damping torque analysis of static VAR system oscillations. IEEE Transactions on Power systems, 1991;6(2):458-65.

[5] P. Kundur, Power System Stability and Control, McGraw-Hill, 1994.

[6] N. G. Hingorani and L. Gyugyi, Understanding FACTS Concepts and Technology of Flexible AC Transmission Systems, New York:IEEE Press, 2000. 
[7] L. Cong, Y. Wang, D.J. Hill "Transient stability and voltage regulation enhancement via coordinated control of generator excitation and SVC" International Journal of Electrical Power \& Energy Systems, Volume 27, Issue 2, pp. 121-130, February 2005.

[8] S. Panda, N. P. Patidar and R. Singh "Simultaneous Tuning of Static Var Compensator and Power System Stabilizer Employing Real-Cod Genetic Algorithm" International Journal of Electrical, Electronic Science and Engineering Vol:2 No:5, 2008.

[9] Gibbard MJ. Robust design of fixed-parameter power system stabilizers over a wide range of operating conditions. IEEE Trans PWRS 1991;6(2):794-800.

[10] Abdel-Magid YL, Abido MA, Al-Baiyat S, Mantawy AH. Simultaneous stabilization of multimachine power systems via genetic algorithms. IEEE PES, Paper \#98 SM 322.

[11]A.S. Bazanella, A.S. e Silva "Coordinated design of damping controllers for robustness of power systems stability" International Journal of Electrical Power \& Energy Systems, Volume 23, Issue 1, pp. 69-79, 1January 2001.

[12] H. Shayeghi, A. Safari, H.A. Shayanfar. PSS and TCSC damping controller coordinated design using PSO in multi-machine power system. Energy Conversion and Management, Volume 51, Issue 12 , pp. 2930-2937, December 2010.

[13]Li-Jun Cai and István Erlich,. Simultaneous Coordinated Tuning of PSS and FACTS Damping Controllers in Large Power Systems. IEEE Trans on POWER SYSTEMS, vol. 20, no. 1, February 2005.

[14] A. Salemnia, M. Khederzadeh, and A. Ghorbani, "Mitigation of Subsynchronous Oscillations by 48-Pulse VSC STATCOM Using Remote Signal"IEEE Bucharest Power Tech Conference, Romania, 2009.

[15] R. Majumder, B. Chaudhuri, B. C. Pal and Q. C. Zhong, A Unified Smith Predictor Approach for Power System Damping Control Design using Remote Signals, IEEE Trans. Control Systems Technology, vol. 13, no. 6, pp. 1063-1068, Nov. 2005.

[16] H. Ghorbani, J. I. Candela, A. Luna, and P. Rodriguez, Posicast Control - A Novel Approach to Mitigate Multi-Machine Power System Oscillations in Presence of Wind Farm, IEEE Power \& Energy Society General Meeting, pp. 1-5, 27 July 2014.

[17] Wei Yao ; Jiang, L. ; Jinyu Wen ; Wu, Q.H. ; Shijie Cheng, Wide-Area Damping Controller of FACTS Devices for Inter-Area Oscillations Considering Communication Time Delays, IEEE Transactions on Power Systems, Vol.29, No.1, pp. 318- 329, 2014.

[18] H. Ghorbani, J. I. Candela, A. Luna, and P. Rodriguez, Application of Posicast control method to generator excitation system, Industrial Electronics (ISIE), 2014 IEEE 23rd International Symposium, pp. 58 - 63.

[19] IEEE Working Group on Prime Mover and Energy Supply Models for System Dynamic Performance Studies, "Hydraulic Turbine and Turbine Control Models for Dynamic Studies," IEEE Transactions on Power Systems, Vol. 7, No.1, pp. 167-179, February, 1992.

[20] A. N. G. Hingorani and L. Gyugyi, Understanding FACTS Concepts and Technology of Flexible AC Transmission Systems, New York: IEEE Press, 2000.

[21] SimPowerSystems Toolbox ver. 5.1, for use with Simulink, User's Guide. The MathWorks, Inc., 2009.

[22] B.Chaudhuri, R.Majumder, B.C. Pal, "Wide-Area Measurement-Based Stabilizing Control of Power System Considering Signal Transmission Delay”, IEEE TRANSACTIONS ON POWER SYSTEMS, VOL. 19, NO. 4, NOVEMBER 2004

[23] http://www.d.umn.edu/ gshute/net/delays-losses.xhtml

[24] M.Hajian, L.Zhang, D.Jovcic, 'DC Transmission Grid With Low-Speed Protection Using Mechanical DC Circuit Breakers', IEEE Transactions on Power Delivery, VOL. 30, NO. 3, June 2015 